\title{
HSP60 is involved in the neuroprotective effects of naloxone
}

\author{
WENJING CHENG $^{1 *}$, YUNHONG LI $^{1 *}$, XIAOLIN HOU ${ }^{2 *}$, NAN ZHANG $^{1}$, JIAO MA $^{1}$, \\ FEIJIA DING ${ }^{1}$, FAN LI ${ }^{1}$, ZHENHUA MIAO ${ }^{1}$, YANLI ZHANG ${ }^{1}$, QI QI ${ }^{1}$, GUANGHUA LI ${ }^{1}$, \\ YING SHEN $^{3}$, JUAN LIU ${ }^{1}$, WEIDONG HUANG ${ }^{1}$ and YIN WANG ${ }^{1}$ \\ ${ }^{1}$ Basic Medical College of Ningxia Medical University, Ningxia Key Laboratory of Cerebrocranial Diseases, Yinchuan, \\ Ningxia 750004; ${ }^{2}$ The General Hospital of Ningxia Medical University, Yinchuan, Ningxia 750004; \\ ${ }^{3}$ Department of Neurobiology, Key Laboratory of Medical Neurobiology of the Ministry of Health, \\ Zhejiang Province Key Laboratory of Neurobiology, Zhejiang University School of Medicine, \\ Hangzhou, Zhejiang 310058, P.R. China
}

Received December 4, 2013; Accepted May 9, 2014

DOI: $10.3892 / \mathrm{mmr} .2014 .2411$

\begin{abstract}
Heat shock protein (HSP)60 is primarily a mitochondrial protein. Previous experiments have found that changes in the location of intracellular HSP60 have been associated with apoptosis. Extracellular HSP60 mediates apoptosis via its ligand, Toll-like receptor (TLR)-4. TLR-4 is an important factor expressed on microglia, with a central role in generating neuroimmune responses in the pathogenesis of neurodegenerative disorders. Naloxone is a highly effective nonselective opioid receptor antagonist, and has been reported to be pharmacologically beneficial for the treatment of brain diseases through inhibiting microglia activation. However, the mechanisms underlying these beneficial effects of naloxone remain poorly understood. The present study aimed to investigate the role of HSP60 in the neuroprotective effects of naloxone on the production of proinflammatory mediators in lipopolysaccharide (LPS)-stimulated BV2 murine microglial cells and the possible signaling pathways involved. The results demonstrated that naloxone significantly inhibited the expression and release of HSP60 in BV2 cells. The expression levels of heat shock factor (HSF)-1 were upregulated in LPS-activated BV2 cells, which indicated that the increased expression of HSP60 was driven by HSF-1 activation. However, increased HSF-1 levels may be downregulated by naloxone. The levels of TLR-4 were elevated in activated
\end{abstract}

Correspondence to: Professor Yin Wang, Department of Neurobiology, Basic Medical College, Ningxia Medical University, Ningxia Key Laboratory of Cerebrocranial Diseases, 1160 Shengli Street, Yinchuan, Ningxia 750004, P.R. China

E-mail: yin-wang@hotmail.com

*Contributed equally

Key words: naloxone, heat shock protein 60, BV2 microglia, Toll-like receptor-4, nuclear factor- $\kappa \mathrm{B}$
BV2 cells, and then inhibited by naloxone. Activation of TLR-4 is characterized by activation of nuclear factor- $\kappa \mathrm{B}$ $(\mathrm{NF}-\mathrm{\kappa B})$ followed by the production of various proinflammatory and neurotoxic factors. Data from the present study demonstrated that naloxone reduced the expression levels of NF- $\mathrm{NB}$ and its upstream protein caspase-3, and reduced the LPS-induced production of nitric oxide, inducible nitric oxide synthase, tumor necrosis factor $\alpha$, interleukin- $1 \beta$ and interleukin-6 in BV2 microglia. In light of this data, it was concluded that naloxone may exert its neuroprotective and anti-inflammatory effects by inhibiting microglia activation through a HSP60-TLR-4-NF-kB signaling pathway.

\section{Introduction}

The activation of microglia, the primary immune cells of the central nervous system (CNS), occurs in almost all neurological disorders (1) and is often associated with the increased production of various pro-inflammatory mediators, including nitric oxide (NO), inducible nitric oxide synthase (iNOS), tumor necrosis factor (TNF)- $\alpha$, interleukin (IL)- $1 \beta$, nuclear factor- $\kappa \mathrm{B}(\mathrm{NF}-\kappa \mathrm{B})$, caspase-3 and heat shock protein (HSP)60 (2-6), which all contribute to neurodegeneration $(7,8)$. The importance of microglial activation in neurodegeneration has prompted speculation that the inhibition of microglial activation, in particular the control of neurotoxic factor production, may be an effective therapeutic option for neurodegenerative diseases. Numerous microglia-targeted pharmacotherapies, including protein kinase $\mathrm{C}$ inhibitors, microglia inhibiting factor, and various Chinese medicinal herb extracts, have been proposed to inhibit the activation of microglia and to promote neuronal survival in vivo (9-11). However, the inability of these drugs to penetrate the blood-brain barrier, in addition to side-effects that they may produce, limit their long-term use in the clinical setting.

Naloxone is a structural analog of morphine and an effective antagonist of the classic opioid receptors that are widely expressed on cells of the central and peripheral nervous systems (12). Administration of naloxone has been demonstrated to be beneficial in the treatment of experimental models 
of stroke, myocardial and brain ischemia, brain trauma, spinal cord injuries and septic shock (13-15). Naloxone has been demonstrated to attenuate the degeneration of dopaminergic neurons by inhibition of $\beta$-amyloid peptide(1-42)-induced microglial activation and degeneration of cortical and mesencephalic neurons, suggesting that naloxone may have potential therapeutic efficacy for the treatment of Parkinson's and Alzheimer's disease $(16,17)$. These studies raise the possibility that naloxone binds to sites other than opioid receptors and exerts activity that does not involve the opioid receptor system. Compared with other microglial inhibitors, naloxone has several advantages, including its ability to penetrate the CNS and that it produces fewer side-effects, presenting a potential novel therapeutic option for neuroprotection.

Although naloxone has been demonstrated to inhibit lipopolysaccharide (LPS)-induced microglial activation in the CNS, the underlying mechanism is not well understood. HSP60 was demonstrated to be released extracellularly in cardiac myocytes during heart failure, and induces apoptosis by binding to Toll-like receptor (TLR)-4 $(18,19)$. The aim of the present study was to examine whether HSP60 is involved in the neuroprotective effects of naloxone in LPS-induced inflammatory injury in BV2 microglia.

\section{Materials and methods}

Chemicals. Naloxone and LPS were purchased from Sigma-Aldrich (St. Louis, MO, USA). Antibodies against $\beta$-actin, NF- $\kappa$ B and TLR-4 (Abcam, Cambridge, MA, USA), anti-HSP60 and anti-heat shock factor (HSF)1 antibodies (Stressgen, San Diego, CA, USA) and an anti-caspase-3 antibody (Cell Signaling Technology, Inc., Beverly, MA, USA) were acquired. Proteinase inhibitor cocktails were purchased from Merck Chemicals (Whitehouse Station, NJ, USA). IL-6, IL-1 $\beta$ and TNF- $\alpha$ ELISA kits were from eBioscience (eBioscience, CA, USA). Bicinchoninic acid (BCA) and enhanced chemiluminescence (ECL) kits were acquired from Pierce (Rockford, IL, USA). Dulbecco's modified Eagle's medium (DMEM) and fetal bovine serum (FBS) came from Gibco (Grand Island, NY, USA). Griess reagent (for identifying NO) and nitric oxide synthase (iNOS) kits were from Jiancheng Bioengineering Institute (Nanjing, China). The Cell Counting Kit-8 (CCK-8) was obtained from Beyotime (Nanjing, China).

Microglial cell culture. BV2 mouse microglia (Cell Bank, Shanghai, China) were cultured in DMEM supplemented with $10 \%$ FBS, penicillin $(100 \mathrm{U} / \mathrm{ml})$ and streptomycin $(100 \mathrm{~g} / \mathrm{ml})$. Cultures were maintained at $37^{\circ} \mathrm{C}$ in a humidified incubator gassed with $95 \% \mathrm{O}_{2}$ and $5 \% \mathrm{CO}_{2}$. Naloxone was dissolved in phosphate-buffered saline (PBS). Cells were treated with the indicated concentrations of naloxone for $24 \mathrm{~h}$ following the administration of LPS $(200 \mathrm{ng} / \mathrm{ml})$ for the indicated time period.

Cell viability assay. Cell viability was measured using the CCK-8. Cells $\left(7.5 \times 10^{3}\right.$ cells in $100 \mu 1$ culture medium/well) were seeded in 96-well plates. CCK-8 solution $(10 \mu \mathrm{l})$ was added to each well and the cultures were incubated at $37^{\circ} \mathrm{C}$ for $90 \mathrm{~min}$. Absorbance at $450 \mathrm{~nm}$ was measured using an immunoreader (Bio-Rad, Beijing, China). The results were plotted as the mean \pm standard deviation of three separate experiments

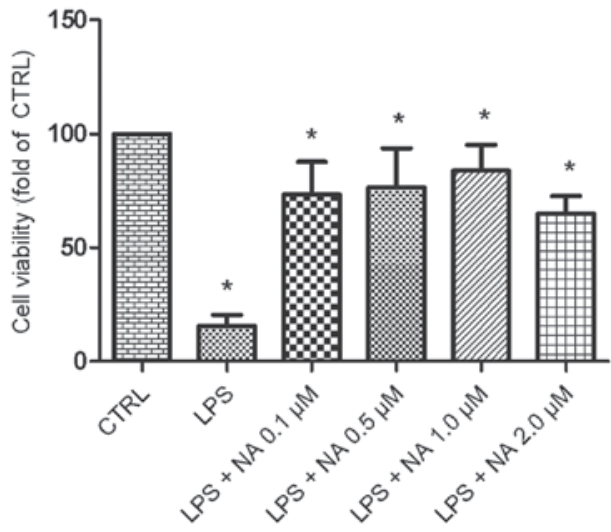

Figure 1. NA increased the cell viability of BV2 microglia. BV2 cells were treated with LPS $(200 \mathrm{ng} / \mathrm{ml})$ for $1 \mathrm{~h}$, followed by treatment with various concentrations of NA $(0,0.1,0.5,1.0$ or $2.0 \mu \mathrm{M})$ for $24 \mathrm{~h}$. The viability of untreated cells was set to 100 . The viability of LPS- and NA-treated cells was expressed as a percentage of the viability of the untreated cells (CTRL). The results are presented as the mean \pm standard error of three separate experiments performed in triplicate, ${ }^{*} \mathrm{P}<0.05$ (LPS group vs. CTRL or NA groups vs. LPS group). CTRL, control; LPS, lipopolysaccharide; NA, naloxone.

having four determinations per experiment for each experimental condition. The cell survival ratio was calculated by normalization to control.

Enzyme-linked immunosorbent assay (ELISA). The levels of IL-6, IL-1 $\beta$ and TNF- $\alpha$ in culture medium were quantified according to the manufacturer's instructions. Absorbance was determined at $450 \mathrm{~nm}$ using a microplate reader (Bio-Rad).

Western blotting. Following treatment, BV2 cells were washed with PBS three times and lysed with radioimmunoprecipitation assay buffer (Sigma-Aldrich). The protein concentration was determined with the BCA kit according to the manufacturer's instructions. Equal quantities of protein were loaded and run on SDS/polyacrylamide gels and then transferred to a polyvinylidine fluoride membrane. Membranes were blocked with $5 \%$ dried milk and incubated with primary antibodies in Tris-buffered saline with Tween 20 overnight at $4^{\circ} \mathrm{C}$. After being rinsed in milk-TBST, blots were incubated in the horseradish peroxidase-conjugated secondary antibodies. The target proteins were detected using the ECL kit and X-ray films (Kodak, Shanghai, China).

Statistical analysis. Statistical significance was determined using one-way analysis of variance. $\mathrm{P}<0.05$ was considered to indicate a statistically significant difference. All data are presented as the mean \pm standard error of the mean.

\section{Results}

Naloxone promotes the viability of BV2 microglia. To determine whether naloxone has an effect on the apoptosis of LPS-stimulated BV2 cells, CCK-8 assay was performed. The results indicated that treatment of microglia with $0.1-2.0 \mu \mathrm{M}$ naloxone for up to $24 \mathrm{~h}$ significantly increased the viability of LPS-stimulated BV2 cells, compared with that of the LPS group (Fig. 1). Naloxone at a concentration of $1.0 \mu \mathrm{M}$ exhibited the maximal protection, so this concentration was selected for 
A

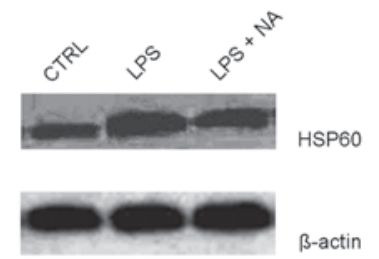

HSP60

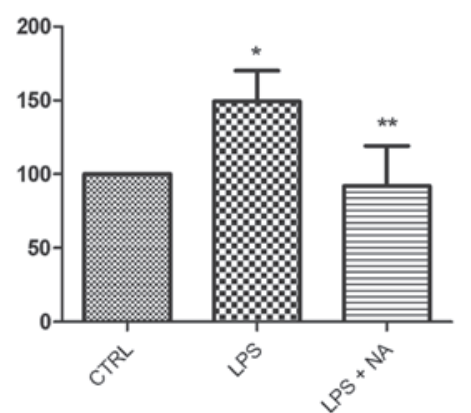

B
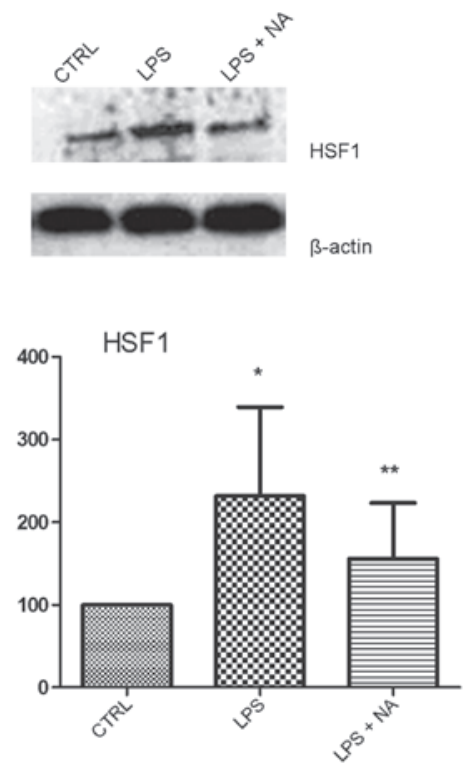

C

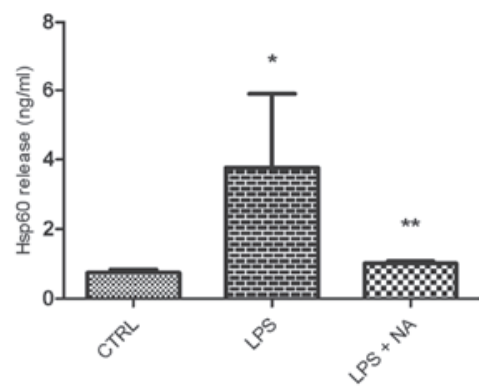

Figure 2. NA inhibited the increased expression and release of HSP60 and HSF-1 in LPS-stimulated BV2 microglia. Cells were pretreated with LPS for 0.5 h, followed by an incubation with $1.0 \mu \mathrm{M}$ NA for $24 \mathrm{~h}$. The lysates were probed by immunoblotting with antibodies against HSP60, HSF-1 and $\beta$-actin. The graphs display ratios of the signal intensities of (A) HSP60/ $\beta$-actin and (B) HSF1/ $\beta$-actin. (C) Extracellular levels of HSP60 were detected by ELISA. Results are presented as the mean \pm standard error of three independent experiments performed in triplicate. ${ }^{*} \mathrm{P}<0.05$ vs. CTRL, ${ }^{* * *} \mathrm{P}<0.05$ vs. LPS group. CTRL, control; LPS, lipopolysaccharide; NA, naloxone; HSP, heat shock protein; HSF, heat shock factor.

the subsequent experiments. The results indicated that naloxone has a positive effect on the viability of LPS-stimulated BV2 microglia.

Naloxone inhibits HSP60 protein expression and release in LPS-stimulated BV2 microglia. Levels of HSP60 expression and release were measured in activated BV2 cells. Western blot results indicated that LPS induced an increase in the expression levels of HSP60 compared with levels in controls, and that naloxone significantly inhibited this increase (Fig. 2A). The HSP60 promoter has been reported to have a heat shock element that is the binding site for HSF-1, which regulates HSP60 gene expression (20). Therefore, HSF-1 levels were investigated, and demonstrated to exhibit the same expression pattern as that of HSP60, indicating that HSP60 expression is driven by HSF-1 (Fig. 2B). HSP60 has been reported to translocate extracellularly upon stress in order to exert injury effects (11). ELISA results demonstrated that HSP60 was released upon LPS-induced activation of BV2 cells, and this increased extracellular HSP60 may be suppressed by naloxone (Fig. 2C).

Naloxone inhibits TLR-4, NF- $\mathrm{BB}$ and caspase-3 expression. TLR-4 mediates inflammatory responses in activated microglia. In the present study, it was demonstrated that TLR-4 expression levels in LPS-stimulated BV2 cells were reduced by naloxone (Fig. 3A). The expression levels of the p65 subunit of NF- $\kappa \mathrm{B}$, which is a pivotal factor in the TLR-4 pathway, increased following LPS stimulation, but this effect was markedly inhibited by the addition of naloxone (Fig. 3B). Caspase- 3 is upstream of $\mathrm{NF}-\kappa \mathrm{B}$ in this signaling pathway, and inhibition of caspase-3 has been demonstrated to prevent neuronal loss in brain diseases involving activated microglia. Thus, in the current study, the effects of naloxone on caspase-3 expression were examined (Fig. 3C). Caspase-3 expression was suppressed in LPS-stimulated BV2 cells following naloxone exposure.

Naloxone inhibits the production of proinflammatory factors. Using ELISA assay, naloxone suppression of the release of proinflammatory factors, including NO, iNOS, TNF- $\alpha$, IL-1 $\beta$ and IL-6, was investigated in LPS-stimulated BV2 cells. As shown in Fig. 4, 24-h naloxone treatment of LPS-stimulated BV2 cells resulted in significant reductions in the levels of the aforementioned factors in culture media compared those following LPS stimulation alone. These results implied that naloxone effectively suppresses the production of neurotoxic factors in activated microglia.

\section{Discussion}

HSP60 is primarily considered to be a mitochondrial protein, but a number of studies have established that HSP60 is also involved in apoptosis. When HSP60 is in the cytosol or mitochondria, it is antiapoptotic and protective. However, when HSP60 is in the plasma membrane or extracellular space, it is associated with apoptosis. Previous studies detected HSP60 expression on the exofacial surface of myocytes, where it was a potential antibody target or innate immune system ligand of TLR-4 $(18,19)$. TLR-4 has been shown to be present in microglia (21), and HSP60 is a ligand for TLR-4 in the immune system (22).

A number of studies have identified neuroprotective effects of naloxone, and it was demonstrated to inhibit LPS-induced microglial activation in the CNS $(16,17)$. However, the mechanisms involved remain unclear. In the present study, it was hypothesized that LPS triggered HSP60 release from microglia, and extracellular HSP60 binds to TLR-4 on the surface of microglia, inducing apoptosis by 
A
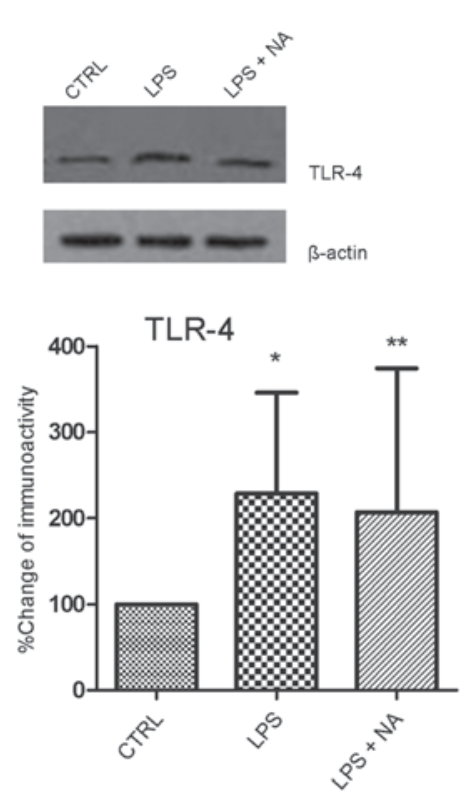

B
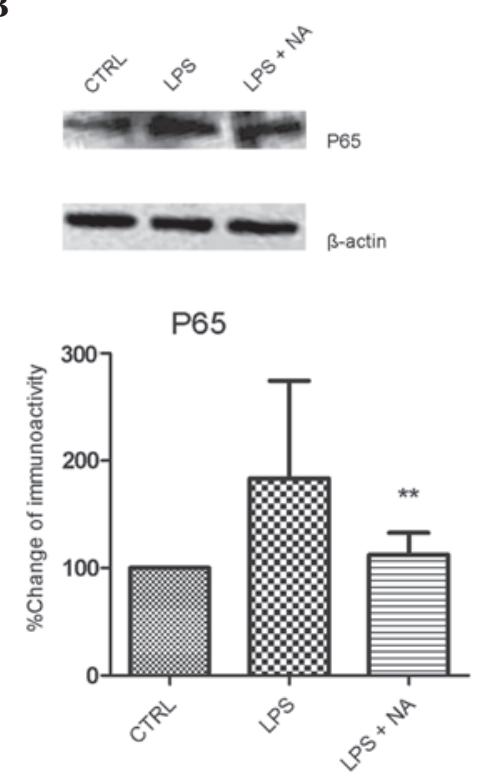

C

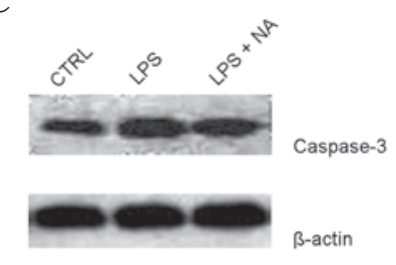

Caspase-3

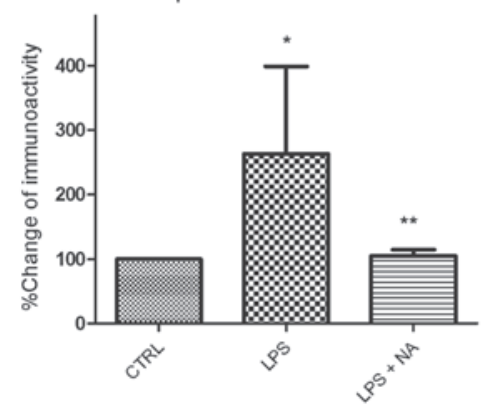

Figure 3. NA inhibited the increased expression of TLR-4, NF- $\kappa$ B and caspase-3 in LPS-stimulated BV2 microglia. Cells were pretreated with LPS for 0.5 h, followed by an incubation with $1.0 \mu \mathrm{M}$ naloxone for $24 \mathrm{~h}$. The lysates were probed by immunoblotting with antibodies against TLR-4, p65, caspase-3 and $\beta$-actin. The graphs display ratios of the signal intensities of (A) TLR-4/ $\beta$-actin, (B) p65/ $\beta$-actin and (C) caspase-3/ $\beta$-actin. Each experiment was derived from at least 6 independent cultures. ${ }^{*} \mathrm{P}<0.05$ vs. CTRL, ${ }^{* *} \mathrm{P}<0.05$ vs. LPS group. CTRL, control; LPS, lipopolysaccharide; NA, naloxone; TLR, Toll-like receptor; $\mathrm{NF}-\kappa \mathrm{B}$, nuclear factor- $\kappa \mathrm{B}$.

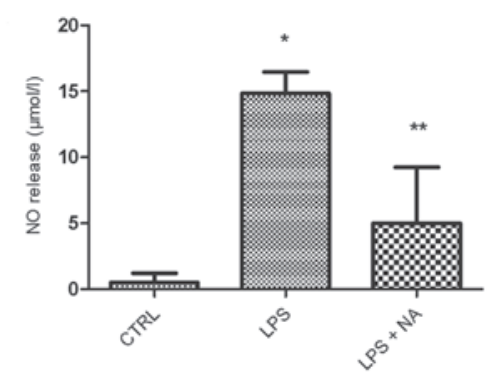

B

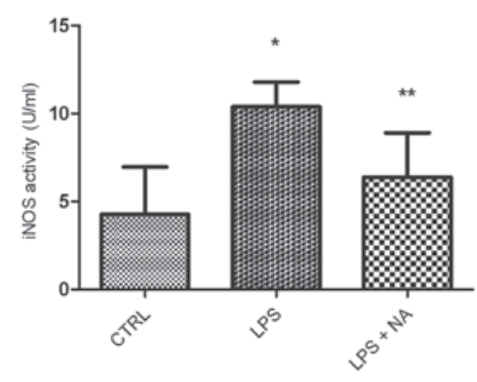

C

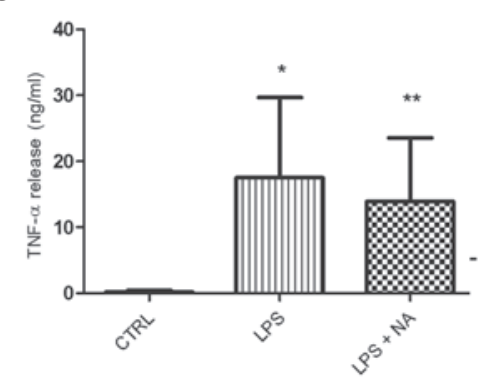

D

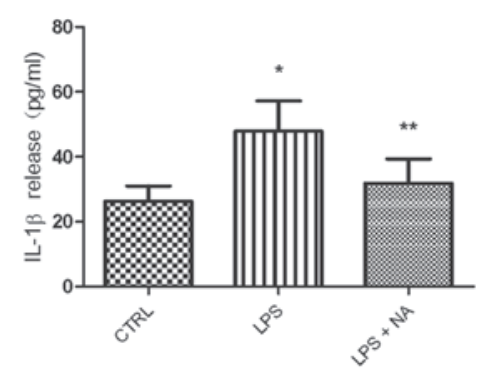

$\mathbf{E}$

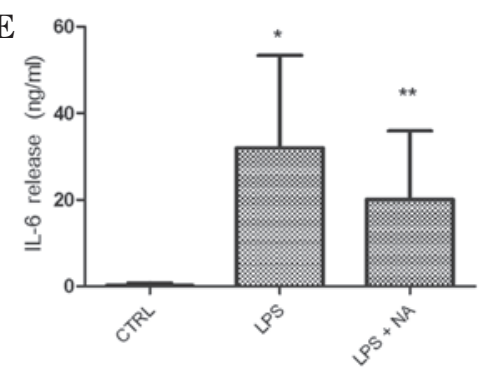

Figure 4. NA reduced the release of NO, iNOS, TNF- $\alpha$, IL-6 and IL-1 $\beta$ in LPS-stimulated BV2 microglia. Cells were pretreated with LPS for $0.5 \mathrm{~h}$, followed by an incubation with $1.0 \mu \mathrm{M}$ NA for $24 \mathrm{~h}$. Extracellular levels of (A) NO and (B) iNOS were assayed with Griess reagent and iNOS kits, respectively, and levels of (C) TNF- $\alpha$, (D) IL-1 $\beta$ and (E) IL-6 were measured using ELISA assay. The results are presented as the mean \pm standard error of three separate experiments performed in triplicate. ${ }^{*} \mathrm{P}<0.05$ vs. CTRL. ${ }^{* * *} \mathrm{P}<0.05$ vs. LPS group. NO, nitric oxide; CTRL, control; LPS, lipopolysaccharide; NA, naloxone; iNOS, inducible nitric oxide synthase; TNF, tumor necrosis factor; IL, interleukin; ELISA, enzyme-linked immunosorbent assay.

activating the NF- $\kappa \mathrm{B}$ pathway. The results supported these hypotheses. A pretreatment of $1 \mu \mathrm{M}$ naloxone effectively suppressed the activation of microglia. While LPS stimulation increased the expression and release of HSP60, naloxone significantly suppressed these effects. HSP expression is regulated by HSFs. In the current study, HSF-1 expression levels increased as did those of HSP60 when triggered by LPS and then were inhibited by naloxone. Thus, the inhibitory effect of naloxone on HSP60 may occur through inhibition of its transcription factor HSF-1. The overexpressed HSP60 
released extracellularly may act as an innate immune signal to further activate microglia.

TLR-4-mediated microglial activation induced cell death, which is a mechanism by which activated immune cells are eliminated. Kim et al (18) demonstrated that extracellular HSP60 activated TLR- 4 by binding to it at a different site to that which LPS binds, leading to cytokine production and cardiac myocytes apoptosis. Release of TNF- $\alpha$ may then lead to further myocyte apoptosis and increased HSP60 expression through activation of NF- $\kappa \mathrm{B}$ (23). In the present study, it was demonstrated that TLR-4 may be activated on microglia not only by LPS but also by extracellular HSP60, and that activated TLR-4 was effectively inhibited by naloxone. LPS and HSP60 binding to TLR-4 may trigger a cascade of signaling events resulting in the activation of downstream effector molecules such as $\mathrm{NF}-\kappa \mathrm{B}$ and caspase 3 and culminating in the production of proimflammatory immune mediators, including TNF- $\alpha$, IL-1 $\beta$, IL-6 and IL-8. Release of NO and chemokines by these cells has also been reported (24). NF- $\kappa \mathrm{B}$ is the major transcription factor in the induction of the transcription of pro-inflammatory genes. The activation of $\mathrm{NF}-\kappa \mathrm{B}$ has been demonstrated to lead to ischemia-induced neuronal injury (25). Caspase-3 is crucial for apoptosis and CNS inflammation (26) and when caspase-3/7 is blocked, activated microglia are non-toxic to neighboring neurons (27). Activation of the NF- $\kappa$ B-p65 cascade induces HSP60 production and release following oxidative stress (19) possibly by the binding of NF- $\kappa$ B to the HSP60 gene promoter (23). TNF- $\alpha$ is also a mediator of $N F-\kappa B$ signaling and drives the increased expression of HSP60, which can be reversed by p65 inhibition (23). Levels of HSP60, NF- $\mathrm{BB}$ and TNF- $\alpha$ are simultaneously decreased by naloxone. By demonstrating the marked inhibition of the expression of caspase- 3 and the NF- $\kappa \mathrm{B}$ downstream mediator $\mathrm{p} 65$, and production of $\mathrm{NO}$, iNOS, TNF- $\alpha$, IL-1 $\beta$ and IL- 6 by naloxone treatment, the findings of the present study suggest that the neuroprotective and anti-inflammatory effects of naloxone may be due to inhibition of the HSP60-TLR-4-NF- $\kappa$ B pathway.

In summary, to the best of our knowledge, the results of the present study indicated for the first time that naloxone may exert its neuroprotective action through HSP60-TLR-4-NF- $\mathrm{B}$ inhibition to prevent the overactivation of microglia.

\section{Acknowledgements}

This study was supported by grants from the National Natural Science Foundation of China (grant nos. 31060140, 31260243, 81060034, 81060126 and 81060112); The Project Sponsored by the SRF for ROCS, State Education Ministry (SEM); The Program for New Century Excellent Talents in University, SEM for Professor Yin Wang; and the Grant of 2012 from Ningxia Medical University for Mr Yunhong Li.

\section{References}

1. Block ML, Zecca L and Hong JS: Microglia-mediated neurotoxicity: uncovering the molecular mechanisms. Nat Rev Neurosci 8: 57-69, 2007.

2. Hanisch UK and Kettenmann H: Microglia: active sensor and versatile effector cells in the normal and pathologic brain. Nat Neurosci 10: 1387-1394, 2007.

3. Gehrmann J, Matsumoto Y and Kreutzberg GW: Microglia: intrinsic immuneffector cell of the brain. Brain Res Rev 20: 269-287, 1995.
4. Innamorato NG, Lastres-Becker I and Cuadrado A: Role of microglial redox balance in modulation of neuroinflammation. Curr Opin Neurol 22: 308-314, 2009.

5. Lynch MA: The multifaceted profile of activated microglia. Mol Neurobiol 40: 139-156, 2009.

6. Li YH, Teng P, Wang Y,Zhang YM, Ma CJ, Pu J,et al: Expression and regulation of HSP60 in activated microglia cells. J Ningxia Med Coll 8: 712-714, 2011.

7. Zhang D, Sun L, Zhu H, Wang L, Wu W, Xie J and Gu J: Microglial LOX-1 reacts with extracellular HSP60 to bridge neuroinflammation and neurotoxicity. Neurochem Int 61: 1021-1035, 2012.

8. Lehnardt S, Schott E, Trimbuch T, Laubisch D, Krueger C, Wulczyn G, Nitsch R and Werber JR: A vicious cycle involving release of heat shock protein 60 from injured cells and activation of toll-like receptor 4 mediates neurodegeneration in the CNS. J Neurosci 28: 2320-2331, 2008.

9. Thanos S, Mey J and Wild M: Treatment of the adult retina with microglia-suppressing factors retards axotomy-induced neuronal degradation and enhances axonal regeneration in vivo and in vitro. J Neurosci 13: 455-466, 1993.

10. Thanos S: The Relationship of Microglial Cells to Dying Neurons During Natural Neuronal Cell Death and Axotomy-induced Degeneration of the Rat Retina. Eur J Neurosci 3: 1189-1207, 1991.

11. Teng P, Li YH, Cheng W, Zhou L, Shen Y and Wang Y: Neuroprotective effects of Lycium barbarum polysaccharides in lipopolysaccharide-induced BV2 microglial cells. Mol Med Rep 7: 1977-1981, 2013.

12. Smith AP and Lee NM: Pharmacology of dynorphin. Annu Rev Pharmacol Toxicol 28: 123-140, 1988.

13. Faden AI and Salzman S: Pharmacological strategies in CNS trauma. Trends Pharmacol Sci 13: 29-35, 1992.

14. Fallis RJ, Fisher M and Lobo RA: A double blind trial of naloxone in the treatment of stroke. Stroke 15: 627-629, 1984

15. Kan MN, Chen YT and Lee AY: Naloxone reversal of ischemic arrhythmia is stereospecific and suggests role of endogenous opioid peptides in ischemic heart disease. Proc Soc Exp Biol Med 200: 518-521, 1992.

16. Liu B, Du L and Hong JS: Naloxone protects rat dopaminergic neurons against inflammatory damage through inhibition of microglia activation and superoxide generation. J Pharmacol Exp Ther 293: 607-617, 2000.

17. Liu Y, Qin L, Wilson BC, An L, Hong JS and Liu B: Inhibition by naloxone stereoisomers of $\beta$-amyloid peptide (1-42)-induced superoxide production in microglia and degeneration of cortical and mesencephalic neurons. J Pharmacol Exp Ther 302: 1212-1219, 2002.

18. Kim SC, Stice JP, Chen L, Jung JS, Gupta S, Wang Y, Baumgarten G, Trial J and Knowlton AA: Extracellular heat shock protein 60, cardiac myocytes, and apoptosis. Circ Res 105: 1186-1195, 2009.

19. Lin L, Kim SC, Wang Y, Gupta S, et al: HSP60 in heart failure: abnormal distribution and role in cardiac myocyte apoptosis. Am J Physiol Heart Circ Physiol 293: H2238-H2247, 2007.

20. Hansen JJ, Bross P, Westergaard M, Nielsen MN, Eiberg H, et al: Genomic structure of human mitochondrial chaperonin genes: HSP60 and HSP10 are localised head to head on chromosome 2 separated by a bidirectional promoter. Hum Genet 112: 71-77, 2003.

21. Aloisi F: Immune function of microglia. Glia 36: 165-179, 2001.

22. Kol A, Lichtman AH, Finberg RW, Libby P and Kurt-Jones EA: Cutting edge: heat shock protein (HSP) 60 activates the innate immune response: CD14 is an essential receptor for HSP60 activation of mononuclear cells. J Immunol 164: 13-17, 2000.

23. Wang Y, Chen L, Hagiwara N and Knowlton AA: Regulation of heat shock protein 60 and 72 expression in the failing heart. J Mol Cell Cardiol 48: 360-366, 2010.

24. Ohashi K, Burkart V, Flohé S and Kolb H: Cutting edge: heat shock protein 60 is a putative endogenous ligand of the toll-like receptor-4 complex. J Immunol 164: 558-561, 2000.

25. Chen J, Zhou Y, Mueller-Steiner S, Chen LF, Kwon H, Yi S, Mucke L and Gan L: SIRT1 protects against microglia-dependent amyloid- $\beta$ toxicity through inhibiting NF- $\kappa \mathrm{B}$ signaling. J Biol Chem 280: 40364-40374, 2005.

26. Soria JA, Arroyo DS, Gaviglio EA, Rodriguez-Galan MC, Wang JM and Iribarren P: Interleukin 4 induces the apoptosis of mouse microglial cells by a caspase-dependent mechanism. Neurobiol Dis 43: 616-624, 2011.

27. Burguillos MA, Deierborg T, Kavanagh E, Persson A, Hajji N, Garcia-Quintanilla A, Cano J, Brundin P, Englund E, Venero JL and Joseph B: Caspase signalling controls microglia activation and neurotoxicity. Nature 472: 319-324, 2011. 